\title{
Determining critical conditions for two dimensional compost piles with air flow via numerical simulations
}

\author{
T. Luangwilai ${ }^{1} \quad$ H. S. Sidhu ${ }^{2}$ \\ (Received 7 December 2010; revised 6 July 2011)
}

\begin{abstract}
We consider the self-heating process of a two dimensional, spatially dependent, model of a compost pile which incorporates terms that account for self-heating due to both biological and oxidative mechanisms. This self-heating model consists of mass balance equations for oxygen and energy. We study the effects of air flow through the pile. Numerical solutions determine critical conditions for thermal ignition within the compost pile. Two distinct numerical approaches corroborate solutions to the self-heating problem in compost piles. We analyse the model numerically using FlexPDE (a commercial software package) in a two dimensional configuration. The results obtained are then compared with those obtained using the Method of Lines. We focus mainly on the critical conditions for thermal ignition for different rates of air flow moving through the compost pile.
\end{abstract}

http://anziamj.austms.org.au/ojs/index.php/ANZIAMJ/article/view/3753 gives this article, (c) Austral. Mathematical Soc. 2011. Published July 27, 2011. ISSN 1446-8735. (Print two pages per sheet of paper.) Copies of this article must not be made otherwise available on the internet; instead link directly to this URL for this article. 


\section{Contents}

1 Introduction

C464

2 Method of lines and FlexPDE

C465

3 Governing equations

C467

3.1 Implementation of the method of lines . . . . . . . . C469

4 Numerical results

C471

4.1 Accuracy . . . . . . . . . . . . . . . . .

$\mathrm{C} 471$

4.2 Comparison of results from MOL and FlexPDE . . . . .

5 Conclusions

$\mathrm{C} 478$

References

C478

\section{Introduction}

Industrial compost piles contain large volumes of bulk organic materials and, typically, two sources of heat generation: the oxidation of cellulosic materials; and biological activity [13]. The first of these represents chemical heat generation and may be modelled by a single Arrehenius reaction [1, 6]. Biological processes that lead to heat generation include the growth and respiration of micro-organisms, such as aerobic mould-fungi and bacteria. This biological heating, which occurs at lower temperatures than oxidation, may increase the temperature to a sufficiently high level to kick-start oxidative reactions. Biological heating is known to be important in large scale composting operations [13] and in the storage of industrial waste fuels, such as municipal solid waste and landfill [8]. Indeed, in composting, self-heating due to biological activity is desirable [2].

Biological activity is known to work efficiently in the elevated temperature 
range of $70-90^{\circ} \mathrm{C}$ which can occur within a few months or even a few days [7]. It has been recognised for almost thirty years that "... biological heating may be an indispensable prelude to self-ignition" [1]. However, mathematical modelling for investigating the spontaneous combustion of compost piles due to biological self-heating is very limited. Sidhu et al. [15] investigated a spatially distributed model for biological self-heating with oxygen consumption but without air flow. Luangwilai et al. [9] extended this model by including air flow through a compost pile in a one dimensional model. We extend this latter study to a two dimensional model with air flow.

In composting, achieving elevated temperatures is desirable. It is at these temperatures that the conditions are ideal for organic breakdown of cellulosic materials [2]. However, fires at composting facilities are not uncommon [13]. Most of these undesirable events have been attributed to thermal ignition of compost piles. Hence determining the conditions for thermal ignition is crucial. However, these critical conditions can only be determined via numerical means. To prevent false predictions due to numerical errors, independent methods must be employed to verify the results.

We report on our use of FlexPDE ${ }^{\mathrm{TM}}$ (a commercial software package) [5] and the Method of Lines (MOL) [14] implemented via MatLab [10] to determine critical conditions for thermal ignition. For completeness, we begin with a brief overview of these two approaches, which is then followed by a discussion regarding the governing equations and the implementation of the numerical schemes. Finally we discuss the accuracy of these methods.

\section{Method of lines and FlexPDE}

FlexPDE $^{\mathrm{TM}}$ is a finite element software package for obtaining numerical solutions to partial differential equations (PDEs). It has the ability to resolve problems in one, two and three dimensions for both steady state and time dependent solutions. It is a space- and time-adaptive finite element pack- 


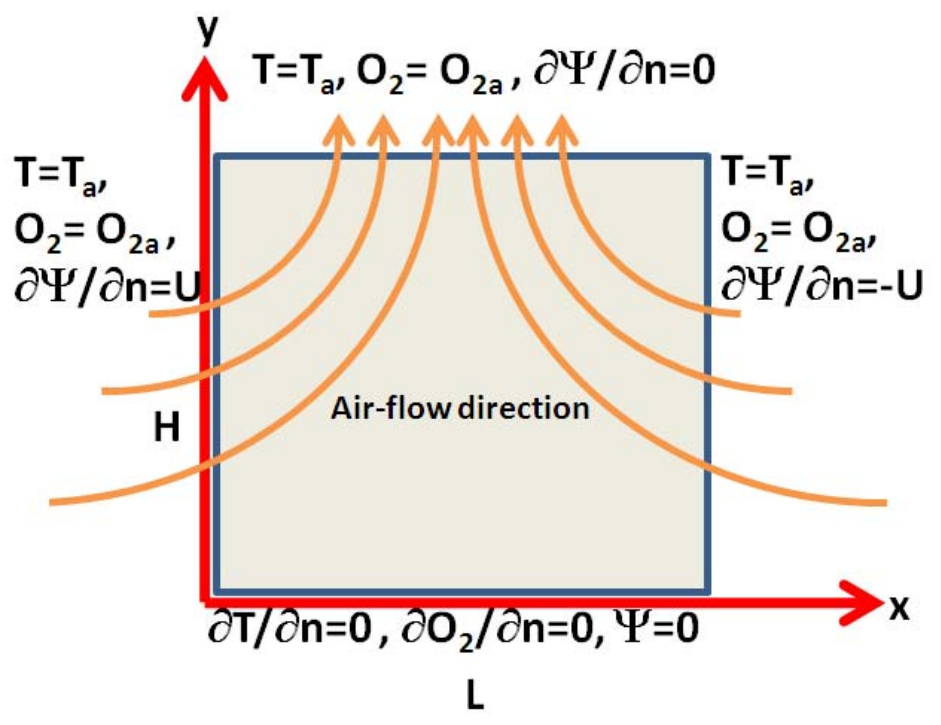

Figure 1: Schematic diagram showing cross-sectional geometry of an infinitely long compost pile and the corresponding boundary conditions.

age which minimises errors to a relative error tolerance level. When using FlexPDE $^{\mathrm{TM}}$, we control the accuracy by setting the value of error tolerance ('ERRLIM' variable in FlexPDE ${ }^{\mathrm{TM}}$ code). If any mesh cell exceeds this tolerance, the scheme refines the mesh size and the solution is recomputed.

The MOL approach is a versatile technique for obtaining numerical solutions to many types of PDEs. The key technique of the MOL is that partial derivatives in spatial directions are replaced by finite difference approximations. In this way, each PDE is replaced by a system of ODEs in time, allowing application of a standard numerical ODE solver. We coded the MOL in MATLAB [10], which has a range of efficient ODE solvers. 


\section{Governing equations}

For simplicity, the geometry of the compost pile is assumed to be an infinite rectangular slab with thickness (width) $\mathrm{L}$ and height $\mathrm{H}$ (see Figure 1). The depletion of cellulosic materials and biomass is ignored. We believe that the effects of temperature gradients and the depletion of cellulosic materials and biomass are negligible for large compost piles for the range of temperatures considered in this study. Moraga et al. [11] showed explicitly, from their experimental data of a sewage sludge pile, that the model and parameter values used by Sidhu et al. [15] (that is, without air flow) provided reasonable predictions of temperature increases within the pile. Since the present model extends the work of Sidhu et al. [15] by including air flow, we believe that the current investigation illustrates the trends when the flow of air is included.

In summary, following the works $[9,15]$, the governing equations contain two equations for the distributions of temperature and oxygen within the pile as follows.

The energy balance on $0 \leqslant x \leqslant L$ and $0 \leqslant y \leqslant H$ is

$$
\begin{aligned}
(\rho C)_{\mathrm{eff}} \frac{\partial T}{\partial t}= & k_{\text {eff }}\left(\frac{\partial^{2} T}{\partial x^{2}}+\frac{\partial^{2} T}{\partial y^{2}}\right)-\epsilon \rho_{\text {air }} C_{a i r}\left(u_{x} \frac{\partial T}{\partial x}+U_{y} \frac{\partial T}{\partial y}\right) \\
& +Q_{c} \rho_{c} A_{3} O_{2}(1-\epsilon) e^{-E_{3} / R T}+Q_{b} \rho_{b}(1-\epsilon) \frac{A_{1} e^{-E_{1} / R T}}{1+A_{2} e^{-E_{2} / R T}}
\end{aligned}
$$

The oxygen balance on $0 \leqslant x \leqslant L$ and $0 \leqslant y \leqslant H$ is

$$
\begin{aligned}
\epsilon \frac{\partial \mathrm{O}_{2}}{\partial t}= & \mathrm{D}_{\text {oeff }}\left(\frac{\partial^{2} \mathrm{O}_{2}}{\partial x^{2}}+\frac{\partial^{2} \mathrm{O}_{2}}{\partial y^{2}}\right)-\epsilon\left(\mathrm{U}_{x} \frac{\partial \mathrm{O}_{2}}{\partial x}+\mathrm{U}_{y} \frac{\partial \mathrm{O}_{2}}{\partial y}\right) \\
& -\rho_{\mathrm{c}} \mathrm{A}_{3} \mathrm{O}_{2}(1-\epsilon) e^{-\mathrm{E}_{3} / \mathrm{RT}} .
\end{aligned}
$$

The second terms on the right-hand sides of equations (1) and (2) model the flow of air through the compost pile, the third terms correspond to the heat generated by the oxidation of cellulosic materials, and the fourth term on the 
right-hand side of equation (1) represents the heat generated by biological activity. The parameters $A_{1}$ and $E_{1}$ in this model represent the increase in the metabolic activity of the biomass at the 'low' temperature range. At sufficiently high temperatures, the essential proteins, which are sensitive to heat, begin to denature and lead to cell death. These processes are represented by the biomass deactivation parameters $A_{2}$ and $E_{2}$. To ensure that the heat release due to biological activity has a global maximum, the activation energy for the inhibition process must be greater than that for the biomass growth, that is, $E_{2}>E_{1}$ (Chen and Mitchell [3] detailed the formulation of the term representing the heat generated by the biomass).

Following the work of Fierro et al. [4], we assume that the air flow directions are as shown in Figure 1. Fierro et al. applied such flows to coal piles. The stream function approach [12] is used to model the air flow and we assume that air flows into the compost pile from the left and right boundaries by natural convection. As the temperature within the pile increases, the air flows up and exits the pile from the top boundary (Figure 1). The stream function describing the air flow is

$$
\frac{\partial^{2} \Psi}{\partial x^{2}}+\frac{\partial^{2} \Psi}{\partial y^{2}}=0 \quad \text { on } 0 \leqslant x \leqslant L \text { and } 0 \leqslant y \leqslant H .
$$

Equation (3) is used to determine the air flow pattern and the air flow rates in the $x$ and $y$ directions for the steady state within the pile. The air flow rates are defined as $U_{x}=\partial \Psi / \partial y$ and $U_{y}=-\partial \Psi / \partial x$. To complete the model, we have the following algebraic relationships:

$$
\begin{aligned}
k_{\text {eff }} & =\epsilon k_{\text {air }}+(1-\epsilon) k_{c}, \\
(\rho C)_{\text {eff }} & =\epsilon \rho_{\text {air }} C_{\text {air }}+(1-\epsilon) \rho_{c} C_{c}, \\
D_{\text {oeff }} & =\epsilon D_{\text {oair }} .
\end{aligned}
$$

The algebraic expressions (4) and (5) define the effective thermal conductivity and the effective thermal capacity of the compost pile in terms of the corresponding properties of air and pure cellulosic material. Equation (6) defines the effective diffusion coefficient for oxygen. 
We also assume that the initial temperature and oxygen distribution within the pile are uniform and equivalent to the ambient conditions. The boundary conditions used in this analysis are shown in Figure 1. All terms that appear in equations (1)-(6) are defined in Table 4 of nomenclature.

\subsection{Implementation of the method of lines}

We briefly overview how we discretised the governing equations and boundary conditions to a form that can be used by the MOL. We consider that variations in $\mathrm{T}, \mathrm{O}_{2}$ and $\Psi$, with respect to $x$ and $y$ are such that the values of $x$ and $y$ are specified in terms of the integer indexes $\boldsymbol{j}$ and $\boldsymbol{i}$ respectively. Therefore, the partial derivative terms are replaced by algebraic second order central space finite difference approximation scheme which evaluates at point $(\mathfrak{j}, \mathfrak{i})$.

We now proceed to discretise the governing equations, (1) and (2), with their respective boundary conditions for both temperature and oxygen concentration. Finite differences approximate all the partial derivative terms on the right-hand sides of equations (1) and (2). However, the derivatives $\partial \mathrm{T} / \partial \mathrm{t}$ and $\partial \mathrm{O}_{2} / \partial t$ are kept. This method leads the governing equations to become a system of ordinary differential equations with only one independent variable $t$ (that is, a system of ODEs) as in expressions (7) and (8).

The energy balance for $1<j<m$ and $1<i<n$ becomes

$$
\begin{aligned}
\frac{d T_{(j, i)}}{d t}= & \frac{k_{\text {eff }}}{(\rho C)_{\text {eff }}}\left(\frac{T_{(j+1, i)}-2 T_{(j, i)}+T_{(j-1, i)}}{\Delta x^{2}}+\frac{T_{(j, i+1)}-2 T_{(j, i)}+T_{(j, i-1)}}{\Delta y^{2}}\right) \\
& -\frac{\epsilon \rho_{\text {air }} C_{a i r}}{(\rho C)_{\text {eff }}}\left(u_{x} \frac{T_{(j+1, i)}-T_{(j-1, i)}}{2 \Delta x}+U_{y} \frac{T_{(j, i+1)}-T_{(j, i-1)}}{2 \Delta y}\right) \\
& +\frac{Q_{c} \rho_{c} A_{3} O_{2(j, i)}(1-\epsilon) e^{-E_{3} / R T_{(j, i)}}}{(\rho C)_{e f f}}+\frac{Q_{b} \rho_{b}(1-\epsilon)}{(\rho C)_{e f f}} \frac{A_{1} e^{-E_{1} / R_{(j, i)}}}{1+A_{2} e^{-E_{2} / R_{(j, i)}}} .
\end{aligned}
$$


The oxygen balance for $1<j<\mathfrak{m}$ and $1<\mathfrak{i}<\mathrm{n}$ becomes

$$
\begin{aligned}
& \frac{\mathrm{dO}_{2(j, \mathrm{i})}}{\mathrm{dt}}=\frac{\mathrm{D}_{\text {oeff }}}{\epsilon}\left(\frac{\mathrm{O}_{2(\mathrm{j}+1, \mathrm{i})}-2 \mathrm{O}_{2(\mathrm{j}, \mathrm{i})}+\mathrm{O}_{2(\mathrm{j}-1, \mathrm{i})}}{\Delta \mathrm{x}^{2}}\right. \\
& \left.+\frac{\mathrm{O}_{2(j, i+1)}-2 \mathrm{O}_{2(j, i)}+\mathrm{O}_{2(j, i-1)}}{\Delta \mathrm{y}^{2}}\right) \\
& -\left(\mathrm{U}_{\mathrm{x}} \frac{\mathrm{O}_{2(\mathrm{j}+1, \mathrm{i})}-\mathrm{O}_{2(\mathrm{j}-1, \mathrm{i})}}{2 \Delta \mathrm{x}}+\mathrm{U}_{\mathrm{y}} \frac{\mathrm{O}_{2(\mathrm{j}, \mathrm{i}+1)}-\mathrm{O}_{2(\mathrm{j}, \mathrm{i}-1)}}{2 \Delta \mathrm{y}}\right) \\
& -\frac{\rho_{c} A_{3} \mathrm{O}_{2}(1-\epsilon) e^{-E_{3} / \mathrm{RT}_{(j, i)}}}{\epsilon} \text {. }
\end{aligned}
$$

From the boundary conditions at the compost base $\left(\partial \mathrm{T} / \partial \mathrm{n}=0\right.$ and $\partial \mathrm{O}_{2} / \partial \mathrm{n}=$ $0)$, the energy and oxygen balance equations are

$$
\frac{d T}{d t}=\frac{k_{\text {eff }}}{(\rho C)_{\text {eff }}}\left(\frac{T_{(j, i+1)}-2 T_{(j, i)}}{\Delta y^{2}}\right) \text { and } \frac{d^{2}}{d t}=\frac{D_{o e f f}}{\epsilon}\left(\frac{O_{2(j, i+1)}-2 O_{2(j, i)}}{\Delta y^{2}}\right) \text {. }
$$

The other boundaries (the two sides and the top), do not need to be transformed and can be written as $\mathrm{T}=\mathrm{T}_{\mathrm{a}}$ and $\mathrm{O}_{2}=\mathrm{O}_{2 \mathrm{a}}$.

Once again we implement the second order central space finite difference approximation for the stream function in equation (3) in order to obtain the air velocities in the $x$ and $y$ directions at steady state conditions. Equation (3) becomes

$$
\Psi_{(j, i)}=\frac{\Delta y^{2}\left(\Psi_{(j, i-1)}+\Psi_{(j, i+1)}\right)+\Delta x^{2}\left(\Psi_{(j-1, i)}+\Psi_{(j+1, i)}\right)}{2\left(\Delta x^{2}+\Delta y^{2}\right)} .
$$

From the boundary conditions of $\partial \Psi / \partial \mathrm{n}=\mathrm{U}$ on the left and right boundaries and $\partial \Psi / \partial \mathrm{n}=0$ on the top boundary, we obtain

$\Psi_{(0, i)}=\Psi_{(2, i)}-2 \Delta x U, \quad \Psi_{(m+1, i)}=\Psi_{(m-1, i)}+2 \Delta x U, \quad \Psi_{(j, n+1)}=\Psi_{(j, n-1)}$. 
TABLE 1: FlexPDE ${ }^{\mathrm{TM}}$ results for maximum steady state temperature within compost pile with different error tolerances (air flow rate at boundaries $\mathrm{U}=10^{-5} \mathrm{~m} \mathrm{~s}^{-1}$ ).

\begin{tabular}{ccccc}
\hline Compost & \multicolumn{4}{c}{ ERRLIM } \\
size $(\mathrm{m})$ & 0.1 & 0.01 & 0.001 & 0.0001 \\
\hline 2 & 298.0333 & 298.0333 & 298.0333 & 298.0333 \\
6 & 298.3081 & 298.3081 & 298.3081 & 298.3081 \\
10 & 298.9099 & 298.9099 & 298.9099 & 298.9099 \\
14 & 299.9932 & 299.9932 & 299.9932 & 299.9932 \\
18 & 302.0725 & 302.0725 & 302.0725 & 302.0725 \\
22 & 368.5891 & 368.5891 & 368.5894 & 368.5894 \\
26 & 373.7847 & 373.7847 & 373.7840 & 373.7840 \\
30 & 379.7441 & 379.7441 & 379.7422 & 379.7422 \\
\hline
\end{tabular}

\section{Numerical results}

In this section, the results from the numerical investigations into the governing equations, (1)-(3), and the corresponding boundary conditions by both FlexPDE ${ }^{\mathrm{TM}}$ and the MOL are presented. The parameter values are based on those previously used by Sidhu et al. [15] and are provided in the nomenclature, Table 4. However, before comparing the results obtained from both methods, the accuracy of the numerical solutions need to be established. In this work, all simulations were run on a desktop PC with a $2.53 \mathrm{GHz}$ Intel xeon (E5540) workstation CPU and 8 Gb memory.

\subsection{Accuracy}

Table 1 shows the FlexPDE ${ }^{\mathrm{TM}}$ results for the maximum steady state temperatures within the compost pile for different compost pile sizes and different error tolerances. The results indicate that when the error tolerances are set to 
0.1 and 0.01 , the steady state temperatures are exactly the same for compost piles of size between 2 to $30 \mathrm{~m}$. However, when the size of the compost pile is greater than $30 \mathrm{~m}$, we find that the results with error tolerances of 0.1 become unreliable since the temperature profile fluctuates randomly, unlike other cases where the temperature profiles increase smoothly. These fluctuations are due to the numerical error of the derivative terms. We also find that the results, when error tolerances are set to 0.001 and 0.0001 , are exactly the same for all compost pile sizes. However, with an error tolerance of 0.0001 , significantly more time is needed to reach the steady state temperature than for ERRLIM $=0.001$. When the error tolerance is reduced to 0.00001 , the results are still exactly the same as those for error tolerances equal to 0.001 and 0.0001 . Consequently, for the remainder of our investigation, we set the error tolerance to 0.001 in order to obtain the most accurate results for this system in the shortest time.

For the MOL approach, we are able to improve the accuracy by increasing the number of node points. The maximum steady state temperature results from the MOL for different compost pile sizes and different numbers of nodes are presented in Table 2, for which the governing equations are integrated over a long period until the temperature reaches a steady state. The number of nodes in the $x$ and $y$ directions are the same which means that $m=n$ (that is, for 400 nodes, $m=n=20$ ). When there are fewer nodes, Matlab takes less time to compute the steady state solution. On the other hand, when the number of nodes is increased, the results become more accurate and more reliable. The results shown in Table 2 for 400, 625, 900 and 1225 nodes are close to each other. However, in our investigation we found that for 400 nodes, the steady state temperature values become unreliable when the temperature is beyond $400 \mathrm{~K}$ (that is, close to the ignition value of $423 \mathrm{~K}$ [13]). At this point, the temperature profile fluctuates randomly. We also observed that when the number of node points is increased to 1600 (40 $\times 40$ nodes), the memory of the computer used to run the MOL scheme in MATLAB becomes insufficient. We find that the results from 900 and 1225 nodes $(30 \times 30$ and $35 \times 35$ nodes) are almost the same (the maximum temperature differences 
TABLE 2: The MOL results for maximum steady state temperature within the compost pile with different number of nodes. Air flow rate at the boundaries are $\mathrm{U}=10^{-5} \mathrm{~ms}^{-1}$.

\begin{tabular}{ccccc}
\hline $\begin{array}{c}\text { Compost } \\
\text { size }(\mathrm{m})\end{array}$ & 400 & 625 & 900 & 1225 \\
\hline 2 & 298.03292 & 298.03308 & 298.03310 & 298.03317 \\
6 & 298.30436 & 298.30589 & 298.30605 & 298.30673 \\
10 & 298.89847 & 298.90314 & 298.90379 & 298.90583 \\
14 & 299.96558 & 299.97639 & 299.97852 & 299.98324 \\
18 & 301.99744 & 302.02307 & 302.03114 & 302.04235 \\
22 & 368.62357 & 368.72899 & 368.71011 & 368.70684 \\
26 & 373.34533 & 373.48428 & 373.51181 & 373.58615 \\
30 & 378.88682 & 379.10607 & 379.24349 & 379.35287 \\
\hline
\end{tabular}

are less than $0.1 \%$ ). However, the case with 1225 nodes takes a significantly longer time for the solutions to achieve the steady state value. As a result, we use 900 nodes $(30 \times 30$ nodes $)$ for the rest of our MOL investigation.

\subsection{Comparison of results from MOL and FlexPDE}

Figure 2 illustrates the results for both numerical schemes for the case when there is no air flow and for the case when the flow is $\mathrm{U}=10^{-5} \mathrm{~m} \mathrm{~s}^{-1}$. Both sets of results predict almost the same temperature values with a maximum difference of less than $0.5 \mathrm{~K}$.

Figure 2(a) shows variations in the maximum steady state temperature within the compost pile as a function of the width of the compost pile (we assume a square configuration) when there is no air flow through the pile. The results from the FlexPDE ${ }^{\mathrm{TM}}$ computations are represented by the red bold curves and those from the MOL by blue circles. Figure 2(a) shows there are two stable solution branches: low temperatures, and elevated temperatures. On the low 


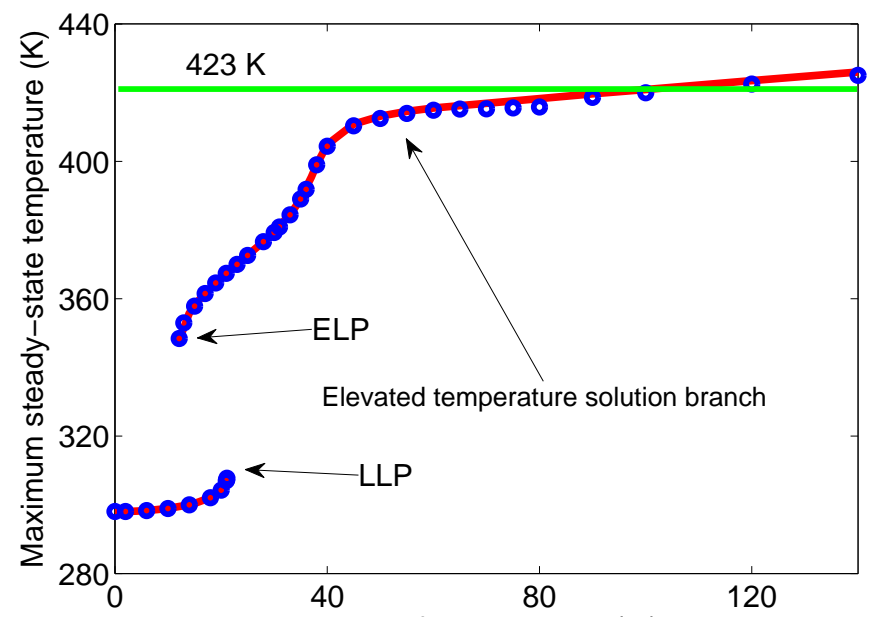

(a)

Width of compost pile $(\mathrm{m})$

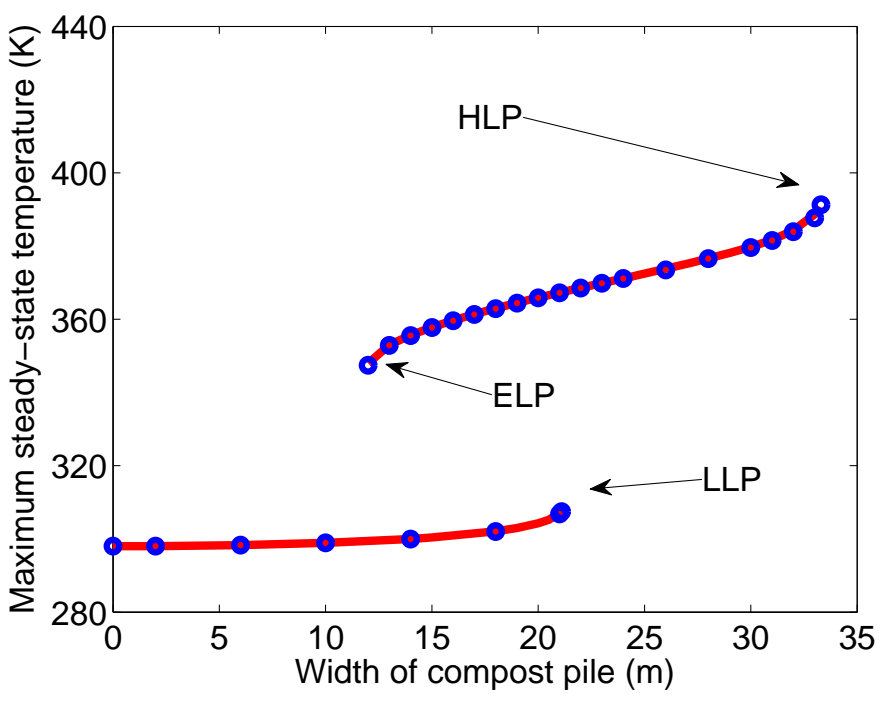

Figure 2: Dependence of maximum steady state temperature within compost heap on size of pile: air flow rate at side boundaries (a) $\mathrm{U}=0 \mathrm{~m} \mathrm{~s}^{-1}$ and (b) $\mathrm{U}=10^{-5} \mathrm{~ms}^{-1}$. LLP refers to low ignition limit point, ELP extinction limit point and HLP high temperature ignition limit point. Red bold curves represent results from FlexPDE ${ }^{\mathrm{TM}}$ and blue circles correspond to the solution from MOL. 
temperature solution branch, the maximum temperature within the pile varies from approximately $298 \mathrm{~K}$ to $308 \mathrm{~K}$. This temperature range is undesirable for composting as it is too low for decomposition processes. FlexPDE ${ }^{\mathrm{TM}}$ predicts the location of the low temperature ignition limit point (LLP) to occur at compost width $\mathrm{L}=21.1 \mathrm{~m}$ with a maximum steady state temperature of approximately $308.26 \mathrm{~K}$. This is very similar to the prediction using MOL-the LLP is located at $L=21.2 \mathrm{~m}$ with a maximum steady state temperature of approximately $308.53 \mathrm{~K}$. When the width of the compost pile is increased beyond the LLP, the steady state solution "jumps" to the elevated temperature solution branch. The critical point on the left-hand side of the elevated temperature solution branch is called the extinction limit point (ELP). FlexPDE ${ }^{\mathrm{TM}}$ predicts the ELP to be located at $\mathrm{L}=12 \mathrm{~m}$ whereas the MOL predicts at $\mathrm{L}=12.5 \mathrm{~m}$. If the width of the compost pile is reduced to a value below that corresponding to the ELP, the solution "drops off" the elevated temperature branch back to the low temperature branch. Between the two limit points (LLP and ELP), there is an unstable solution branch which cannot be detected in our numerical schemes since integration of the governing differential equations with the associated boundary and initial conditions does not yield unstable solutions. As a result, the solution branches appear to be disjointed.

In the steady state plot in Figure 2(a), it can be seen that, at the beginning of the elevated temperature solution branch, the maximum steady state temperature within the compost pile increases rapidly as the width of the compost pile is increased. The rate of the temperature rise decreases significantly after the maximum steady state temperature reaches $410 \mathrm{~K}$. This makes physical sense since, along the elevated temperature branch, both the oxidative and biological reactions generate heat. However, once the maximum temperature becomes sufficiently high, the contribution of biological self-heating decreases as the micro-organisms start to die (or become dormant). The contribution of chemical heating also decreases as there is insufficient oxygen within the pile. As a result of these mechanisms, the rate of change in the maximum temperature is dramatically reduced.

When there is no air flow, the steady state diagram Figure 2(a) illustrates 
two notable features. Firstly, the maximum steady state temperature on the elevated temperature solution branch gradually increases as the width of the compost pile is increased. Since compost materials generally ignite at about $423 \mathrm{~K}\left(150^{\circ} \mathrm{C}\right)$ [13], it is clear that, in this case, ignition only occurs for very large piles, that is, for width and height greater than $100 \mathrm{~m}$. Secondly, the transition from the elevated temperature solution to the flaming combustion solution is "smooth" and not characterised by a bifurcation. From the operational viewpoint, the elevated temperature branch is desirable since the composting process is enhanced at such temperatures. However, ignition of the compost pile must also be avoided.

Next, results of the investigations into the effects of air flow through a two dimensional square compost pile are presented. For low air flow rates, the steady state solutions resemble those shown in Figure 2(a). When the flow rate is fixed at $\mathrm{U}=10^{-5} \mathrm{~m} \mathrm{~s}^{-1}$, the solutions bahaviour changes from that seen in Figure 2(a), namely the solution branch "breaks' into two short solution branches. This is shown in Figure 2(b). Besides the LLP and ELP seen in Figure 2(a), there now exists another limit point which is the high temperature ignition limit point (HLP). The elevated temperature solution branches are significantly shorter than before. Choosing a value of the compost width greater than the HLP value results in a big "jump" in temperature which represents spontaneous ignition within the pile as the temperature increases beyond the ignition value of $423 \mathrm{~K}$. This is because, due to the air flow, there is now sufficient oxygen for the oxidative reaction to generate heat inside the compost pile. Therefore, as all the limit points, particularly HLP, are located at the smaller pile size, the chance of spontaneous ignition is now more likely to occur with a small compost pile. In order to achieve the desirable temperature solution branch, the width of the compost pile must be greater than the LLP but, to prevent spontaneous ignition, less than the HLP. Therefore, from an operational point of view, the locations of LLP and HLP are more important than the ELP. As in the earlier case, Figure 2(b) also shows that both the FlexPDE ${ }^{\mathrm{TM}}$ and MOL schemes predict almost identical solution behaviour. 
TABLE 3: Table showing locations of critical points (in metres) using different numerical methods and air flow rates (LLP refers to low temperature ignition limit point and HLP to high temperature ignition limit point).

\begin{tabular}{|c|cc|cc|}
\hline Air speed & \multicolumn{2}{|c|}{ MOL } & \multicolumn{2}{c|}{ FlexPDE } \\
$\left(\mathrm{m} \mathrm{s}^{-1}\right)$ & LLP & HLP & LLP & HLP \\
\hline 0 & 21.2 & - & 21.1 & - \\
$10^{-5}$ & 21.1 & 33.3 & 21.1 & 33.2 \\
$10^{-4}$ & 21.8 & 35.0 & 22.2 & 35.3 \\
$10^{-3}$ & 82 & n/a & 83.5 & 130.0 \\
\hline
\end{tabular}

Table 3 shows the locations of the LLP and HLP for various values of the air flow rate using MOL and FlexPDE ${ }^{\mathrm{TM}}$. Again, both numerical methods predict very similar values of the LLP and HLP for the width of the compost pile. As previously mentioned, the HLP does not exist in the case with no air flow. However, when the air flow rate is increased to $10^{-5} \mathrm{~m} \mathrm{~s}^{-1}$ or $10^{-4} \mathrm{~m} \mathrm{~s}^{-1}$, the chance of spontaneous ignition is also increased since the HLP can be achieved for a smaller compost pile - there is sufficient oxygen being supplied from the surroundings which enhances the oxidative reaction therefore creating more heat. On the other hand, when the air flow rate is increased further to $10^{-3} \mathrm{~m} \mathrm{~s}^{-1}$, we find that both the LLP and HLP are located at very large pile sizes, $83.5 \mathrm{~m}$ and $130.0 \mathrm{~m}$ respectively, according to the results obtained using FlexPDE ${ }^{\mathrm{TM}}$. The reason that the LLP and HLP are located at very large pile sizes is that the cooling mechanism from the air flow dominates the heat generation process. The MOL approach could only detect the LLP, as there was insufficient memory on the workstation to compute the solutions to determine the location of the HLP for large compost piles. 


\section{Conclusions}

We investigated the spontaneous ignition of the compost heaps using two independent numerical approaches: the MOL scheme implemented using MATLAB, and the finite element scheme via the use of the commercial software FlexPDE $^{\mathrm{TM}}$. The results obtained using FlexPDE ${ }^{\mathrm{TM}}$ and the MOL are found to be almost identical, with the maximum steady state temperature difference being less than $0.5 \mathrm{~K}$. We believe that to prevent false predictions, it is important to verify results using independent approaches when only numerical means are available, as was in this case. These two independent methods corroborate the solution behaviour found in this problem, particularly the phenomenon of thermal ignition.

From both numerical schemes, it has been shown that the value of the air flow velocity is crucial for determining the behaviour of a compost pile. It acts both to increase the rate of heat generation by supplying oxygen for oxidative reaction and to decrease it by removing heat from the compost pile. When the velocity is too high, heat loss dominates and very large pile sizes are required to reach the desired temperature. When the velocity is too low, there is insufficient oxygen within the pile to generate high temperatures and, as a result, internal heat generation from the oxidative reaction ceases at elevated temperatures. For intermediate values of the air flow rate, elevated temperatures can be achieved by moderately sized compost piles. However, this gain in performance is counterbalanced by the possibility that the compost pile will spontaneously ignite.

\section{References}

[1] P. C. Bowes, Self-heating: Evaluating and Controlling the Hazards, Elsevier Press, Amsterdam, The Netherlands, 1984. C464, C465 


\section{TABLE 4: nomenclature.}

$A_{1} \quad$ Pre-exponential factor for oxidation of biomass growth $\left(2.0 \times 10^{6} \mathrm{~s}^{-1}\right)$

$A_{2} \quad$ Pre-exponential factor for inhibition of biomass growth $\left(6.86 \times 10^{30}\right)$

$A_{3}$ Pre-exponential factor for oxidation of cellulosic material $(1.8 \times$ $10^{4} \mathrm{~m}^{3} \mathrm{~kg}^{-1} \mathrm{~s}^{-1}$ )

$\mathrm{C}_{\text {air }} \quad$ Heat capacity of air $\left(1005 \mathrm{~J} \mathrm{~kg}^{-1} \mathrm{~K}^{-1}\right)$

$\mathrm{C}_{\mathrm{c}} \quad$ Heat capacity of cellulosic material $\left(3320 \mathrm{~J} \mathrm{~kg}^{-1} \mathrm{~K}^{-1}\right)$

$\mathrm{D}_{\text {oair }}$ Diffusion coefficient for oxygen $\left(10^{-5} \mathrm{~m}^{2} \mathrm{~s}^{-1}\right)$

$E_{1} \quad$ Activation energy for biomass growth $\left(10^{5} \mathrm{~J} \mathrm{~mol} \mathrm{biomass}^{-1}\right)$

$E_{2}$ Activation energy for inhibition of biomass growth $(2 \times$ $10^{5} \mathrm{~J} \mathrm{~mol} \mathrm{biomass}^{-1}$ )

$E_{3}$ Activation energy for oxidation of cellulosic material $(1.1 \times$ $10^{5} \mathrm{~J} \mathrm{~mol}^{-1}$ )

$\mathrm{L}, \mathrm{H} \quad$ Width and height of compost pile respectively $(\mathrm{m})$

$\mathrm{O}_{2} \quad$ Oxygen concentration within pile $\left(\mathrm{kg} \mathrm{m}^{-3}\right)$

$\mathrm{O}_{2 \mathrm{a}} \quad$ Ambient oxygen concentration $\left(0.272 \mathrm{~kg} \mathrm{~m}^{-3}\right)$

$\mathrm{Q}_{\mathrm{b}} \quad$ Exothermicity for oxidation of biomass per $\mathrm{kg}$. of cellulose $(6.6 \times$ $10^{6} \mathrm{~J} \mathrm{~kg}^{-1}$ )

$\mathrm{Q}_{\mathrm{c}} \quad$ Exothermicity for oxidation of cellulosic material $\left(1.7 \times 10^{7} \mathrm{~J} \mathrm{~kg}^{-1}\right)$

$\mathrm{R} \quad$ Ideal gas constant $\left(8.314 \mathrm{~J} \mathrm{~K}^{-1} \mathrm{~mol}^{-1}\right)$

$\mathrm{T}$ Temperature within compost pile (K)

$\mathrm{T}_{\mathrm{a}} \quad$ Ambient temperature $(298 \mathrm{~K})$

$\mathrm{U} \quad$ Air velocity through the pile $\left(\mathrm{m} \mathrm{s}^{-1}\right)$

$k_{\text {air }} \quad$ Effective thermal conductivity of air $\left(0.026 \mathrm{Wm}^{-1} \mathrm{~K}^{-1}\right)$

$k_{c} \quad$ Effective thermal conductivity of cellulose $\left(0.3 \mathrm{Wm}^{-1} \mathrm{~K}^{-1}\right)$

$\mathrm{t} \quad$ Time $(\mathrm{s})$

$x, y$ Spatial distance along the width and the height of pile respectively (m)

$\epsilon \quad$ Void fraction (0.3)

$\Psi \quad$ Stream function

$\rho_{\text {air }} \quad$ Density of air $\left(1.17 \mathrm{~kg}, \mathrm{~m}^{-3}\right)$

$\rho_{\mathrm{b}} \quad$ Density of bulk biomass within compost pile $\left(0.5 \times \rho_{\mathrm{c}} \mathrm{kg} \mathrm{m}^{-3}\right)$

$\rho_{c} \quad$ Density of pure cellulosic material $\left(1150 \mathrm{~kg} \mathrm{~m}^{-3}\right)$ 
[2] W. F. Brinton, E. Jr. Evans, M. L. Droffner and R. B. Brinton, Standardized test for evaluation of compost self-heating, BioCycle, 36, 60-65, 1995. http://www. jgpress.com C464, C465

[3] X. D. Chen and D. A. Mitchell, Start-up strategies for self-heating and efficient growth in stirred bioreactors for solid state fermentation, In Proceeding of the 24th Australian and New Zealand Chemical Engineering Conference, 4, The Institution of Engineers, 111-116, 1996. C468

[4] V. Fierro, J. L. Miranda, C. Romero, J. M. Andres, A. Arriaga and D. Schamal, Model predictions and experimental results on self-heating prevention of stockpiled coals, FUEL, 80 (2008), 125-134, 2001. doi:10.1016/S0016-2361(00)00062-4 C468

[5] FlexPde, PDE Solutions Inc, http://www.pdesolutions.com. C465

[6] D. A. Frank-Kamenetskii, Diffusion and Heat Transfer in Chemical Kinetics, 2nd edition, Plenum Press, New York, USA, 1969. C464

[7] W. Hogland, T. Bramryd and I. Persson, Physical, biological and chemical effects of unsorted fractions of industrial solid waste fuel storage, Waste Manage. Res., 32, 21-98, 1996. doi:10.1006/wmre.1996.0019 C465

[8] P. F. Hudak, Spontaneous combustion of shale spoils at sanitary landfill, Waste Manage., 22, 687-688, 2001. doi:10.1016/S0956-053X(01)00077-0 $\mathrm{C} 464$

[9] T. Luangwilai, H. S. Sidhu, M. I. Nelson and X. D. Chen, Airflow and ambient temperature effects on the biological self-heating of compost piles, Asia-Pac. J. of Chem. Eng., 5(4), 609-618, 2010. doi:10.1002/apj.438 C465, C467

[10] Matlab, MathWorks Inc, http://www . mathworks.com. C465, C466 
[11] N. O. Moraga, F. Corvalan, M. Escudey, A. Arias and C. E. Zambra, Unsteady 2D coupled heat and mass transfer in porous media with biological and chemical heat generations, Int. J. Heat Mass Tran., 52, 5841-5848, 2009. doi:10.1016/j.ijheatmasstransfer.2009.07.027 C467

[12] V. D. Naylor, The stream function and the velocity potential function, Math. Gaz., 38(323), 30-34, 2007. http://www.jstor.org/stable/3609771 C468

[13] R. Rynk, Fires at composting facilities, BioCycle Magazine, 41(1), 54-58, 2000. http://www.jgpress.com C464, C465, C472, C476

[14] W. E. Schiesser, The numerical method of lines: Integration of Partial Differential Equations, Academic Press, Inc, 1991. C465

[15] H. S. Sidhu, M. I. Nelson and X. D. Chen, A simple spatial model for self-heating compost piles, ANZIAM J(E), 48, C135-C150, 2007, http://anziamj . austms.org.au/ojs/index.php/ANZIAMJ/article/ view/86 C465, C467, C471

\section{Author addresses}

1. T. Luangwilai, Applied and Industrial Mathematics Research Group, School of Physical, Environmental and Mathematical Sciences, University of New South Wales, Canberra, ACT 2600, Australia. mailto:t.luangwilai@student.adfa.edu.au

2. H. S. Sidhu, Applied and Industrial Mathematics Research Group, School of Physical, Environmental and Mathematical Sciences, University of New South Wales, Canberra, ACT 2600, Australia. 